\title{
Simultaneous Echo Refocused Magnetization Transfer Imaging
}

\author{
Claus Kiefer \\ Support Center for Advanced Neuroimaging (SCAN), Institute for Diagnostic and Interventional Neuroradiology, \\ University Hospital Inselspital and University of Bern, Bern, Switzerland \\ Email: claus.kiefer@insel.ch
}

Received 16 July 2014; revised 16 August 2014; accepted 15 September 2014

Copyright (C) 2014 by author and Scientific Research Publishing Inc.

This work is licensed under the Creative Commons Attribution International License (CC BY).

http://creativecommons.org/licenses/by/4.0/

(c) $\underset{\mathrm{EY}}{\mathrm{B}}$ Open Access

\begin{abstract}
Purpose: To increase the efficiency of densely encoded magnetization transfer imaging of the brain, we time-multiplex multiple slices within the same readout using simultaneous echo refocusing FLASH imaging with magnetization transfer (MT) preparation (MT-SER-FLASH). Materials and Methods: Inefficiency in total scan time results from the number of frequency samples needed for sufficient quality of quantitative parameter maps for a binary spin bath model. We present a highly efficient multiplexing method, simultaneous echo refocused magnetization transfer imaging (MT-SER-FLASH) for reducing the total scan time of MT imaging by one-third. The specific absorption rate (SAR) was also reduced by reducing the number of MT-pulses per volume. Results: 2D-MT-SER-FLASH is performed in 19 minutes rather than 1 hour, acceptable for routine clinical application. The SAR could be reduced to $69 \%$ instead of more than $100 \%$ with a standard $2 D$ or 3D-FLASH with MT-preparation. Conclusion: The net reduction of scan time and SAR enables the use of quantitative model based magnetization transfer imaging within a clinical environment.
\end{abstract}

\section{Keywords}

MRI, Magnetization Transfer Imaging, Fast Imaging, Pulse Sequences, Simultaneous Echo Refocusing

\section{Introduction}

Beside T1 and T2 relaxation time, the magnetization transfer (MT) contrast [1]-[3] is a diagnostic relevant parameter as shown in [4]. One question that give rise for further optimization of model based quantitative approaches in clinical diagnostics is for example, if model based MT (mMT), in contrast to MTR (MT ratio), is capable to differentiate different types of macromolecules by their interaction with the environment (free pool) 
and the related parameters such as transfer rates or the T2 time of the restricted pool. A first step towards the solution of this issue will be investigated within the scope of this technical note and will consist in the implementation of a suitable mMT MR-sequence. Beside approaches such as Cramer-Rao lower bounds (CRLB) to obtain optimal acquisition schemes [5], which are not discussed within this note, the focus is on the acceleration of mMT sequences covering an off-frequency range from 500 to $16000 \mathrm{~Hz}$ with a step size $\Delta f$ less than $900 \mathrm{~Hz}$. Against this background in order to acquire full brain data at e.g. 20 different frequency offsets $(\Delta f=816 \mathrm{~Hz})$ it is necessary to keep the acquisition time within an clinically acceptable range of less than 20 minutes-combined with T1 and T2 relaxometry we then end up with a 30 minutes protocol. With the recently proposed simultaneous echo refocusing approach [6]-[10] a modified FLASH sequence with MT preparation was implemented which enables at least a factor three of acceleration. The sequence was tested for a phantom with an $8 \%$ agar concentrate.

\section{Methods and Materials}

The sequence is a modified 2D-FLASH sequence as shown in Figure 1. After the MT preparation pulse (flip angle 540 degree) and a spoiler in z direction three echoes from three different slices will be generated by the three subsequent rf-pulses (flip angle 15 degree) during one acquisition. The saved time can be used to acquire more frequency samples. The exemplary phase graph shows how switching the defocusing gradients $\mathrm{G}_{\mathrm{d}} 1-3$ and readout gradient $G_{r}$ which cause the signals of the $S 1$ - 3 magnetization pathways refocusing at null points of phase (Figure 2). The sequence parameters are as follows: MB-factor 3, SAR-factor 2, 60 slices, gap 0, voxel size $=2 \mathrm{~mm}$ isotropic, 20 frequency samples, matrix size $=128, \mathrm{TR} / \mathrm{TE}=30 / 4.8 \mathrm{~ms}, \mathrm{BW}=390 \mathrm{~Hz} / \mathrm{Px}, \mathrm{TA}=19$ min 12 sec. The defocusing gradients are calculated, $\mathrm{m}_{\mathrm{k}}=0.01 * \mathrm{~s}_{\mathrm{k}}{ }^{*} \mathrm{G}_{\mathrm{RO}} *\left(\Delta_{\mathrm{ADC}}+\Delta_{\mathrm{ramp}}\right)(\mathrm{k}=0.2)$, where $\mathrm{m}$ is the needed moment for dephase, the $s$ are for scaling the $G_{d} 1-3$ amplitudes, $G_{R O}$ is the amplitude of the readout gradient, $\Delta_{\mathrm{ADC}}$ is the duration of the ADC period and $\Delta_{\mathrm{ramp}}$ is the ramp-up time of the readout gradient. The SAR factor determines how often the MT-preparation pulse is acquired: SAR = 2 e.g. means, that only every second $k$-space line the pulse is applied. The scaling factors $\mathrm{s}_{\mathrm{k}}$ are determined by checking for optimal image quality and minimal phase distortions (see Figure 3, Figure 4). The SAR value itself is given in percent of the limit on clinical whole body scanners.

Imaging was performed on a 3.0 Tesla whole body scanner. The phantom was a cylinder (13 cm diameter, 10 $\mathrm{cm}$ height), filled with water and four integrated tubes filled with agar gel (2\%, $4 \%, 8 \%)$ and manganese chloride $0.3 \mathrm{mM}$ respectively.

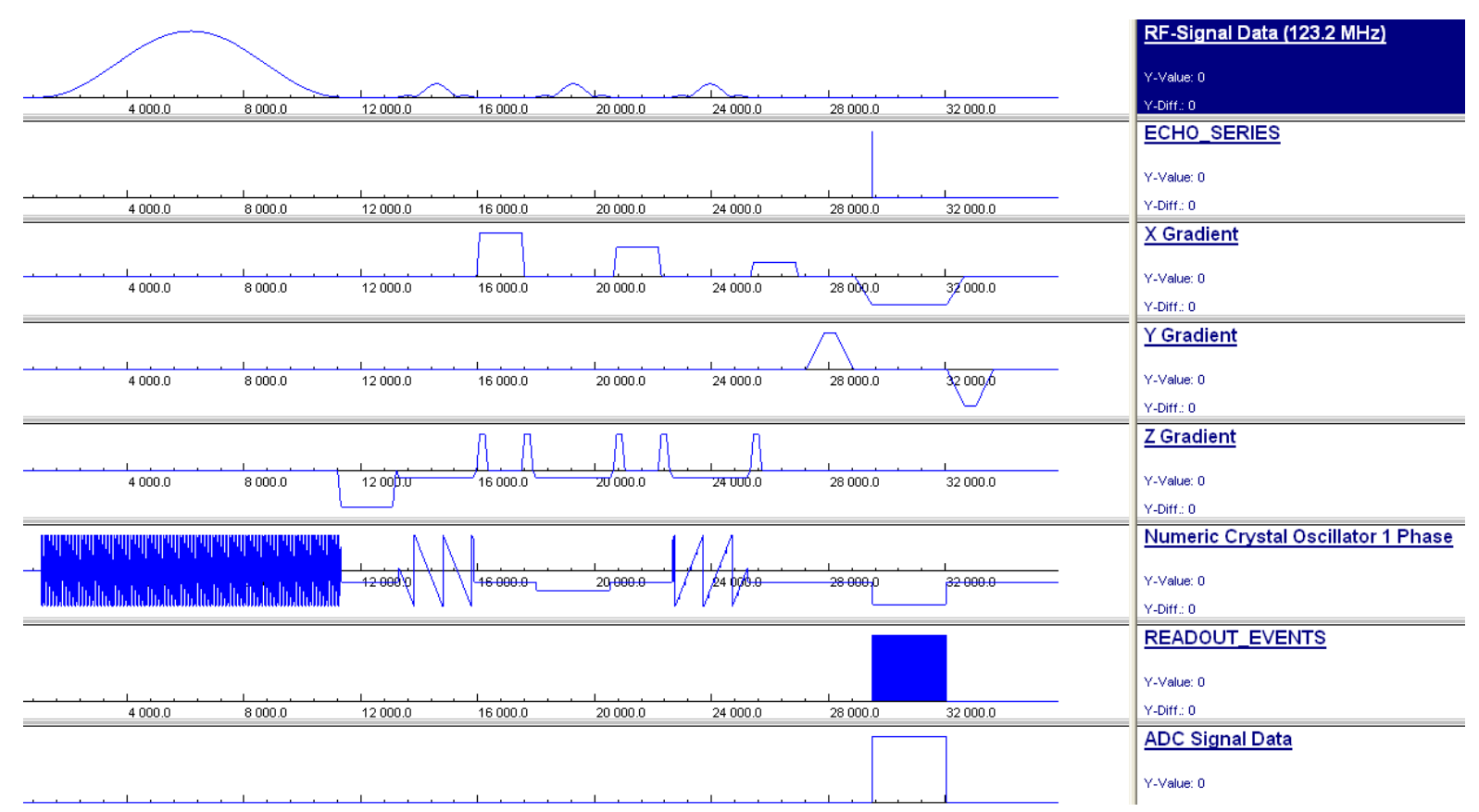

Figure 1. MT-SER-FLASH. Defocusing and readout gradients shown in row denoted as “X Gradient”. 


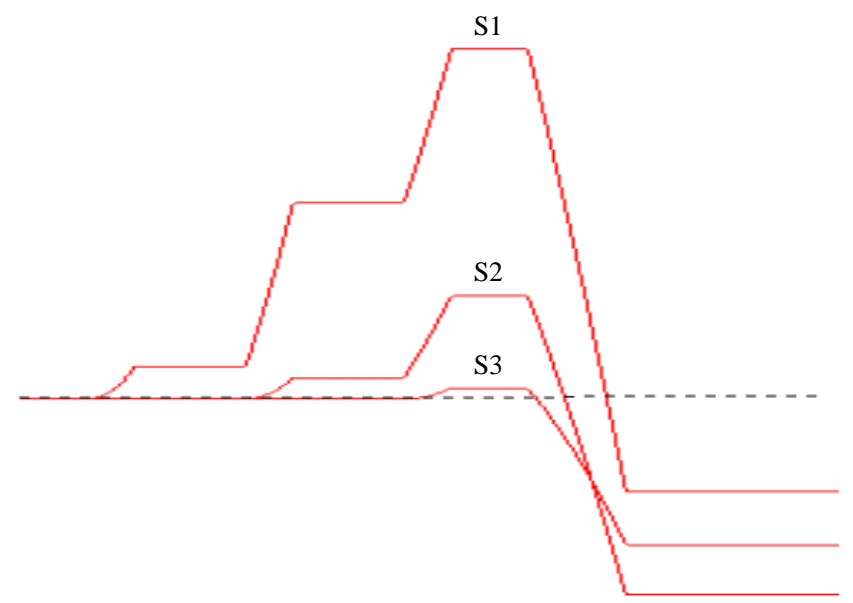

(a)

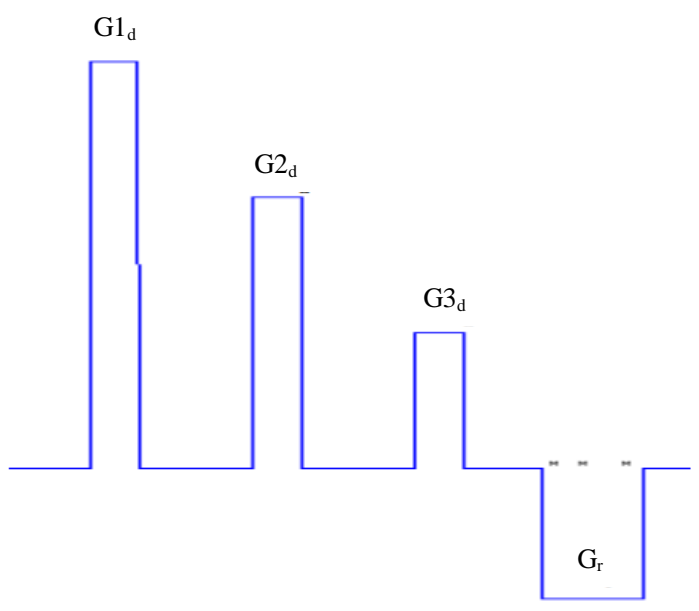

(b)

Figure 2. Phase graph (a) and echo positions (b). Defocusing gradients $G_{d} 1-3$ and readout gradient $G_{r}(b)$ cause the signals of the S1 - 3 magnetization pathways (a) refocusing at null points (marked as *) of the phase (b).

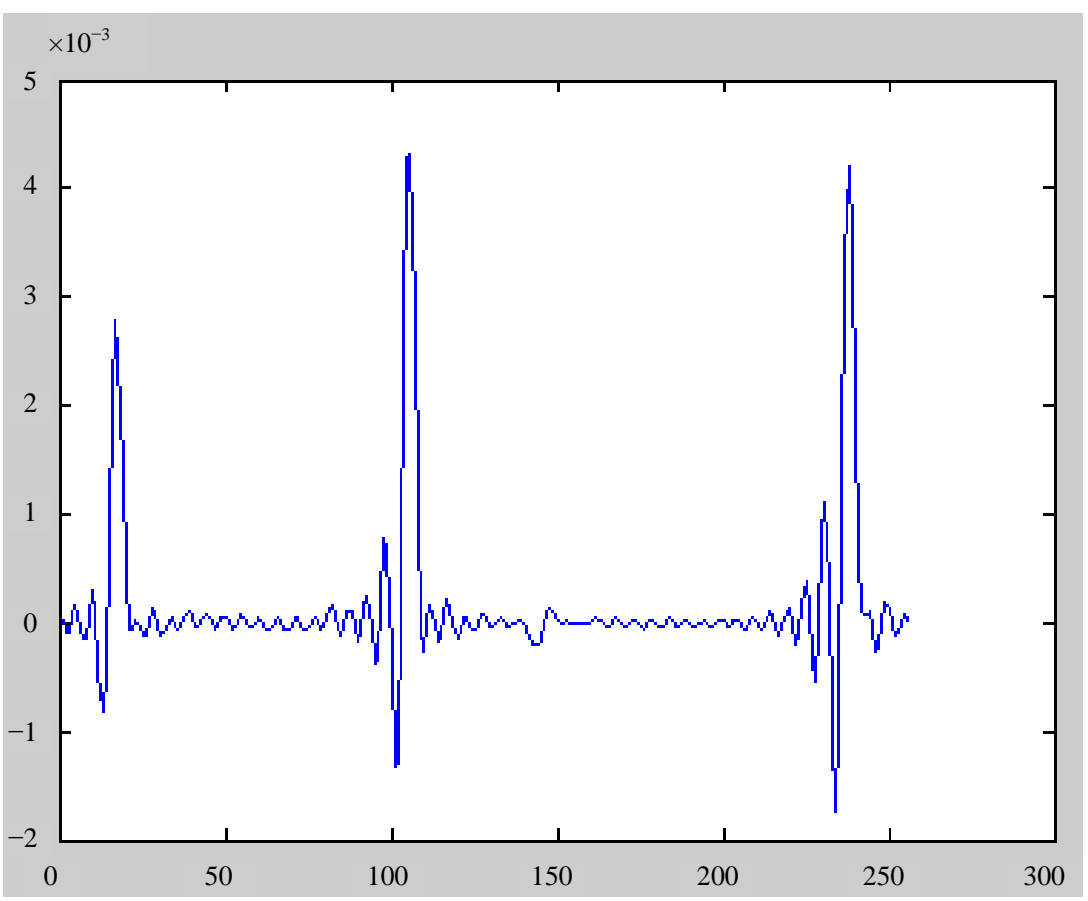

Figure 3. Check of the relative echo positions and their quality (without MT-pulse).

$\Delta f=16000$

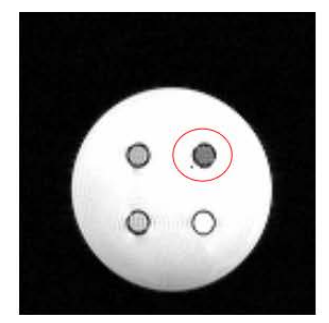

12125

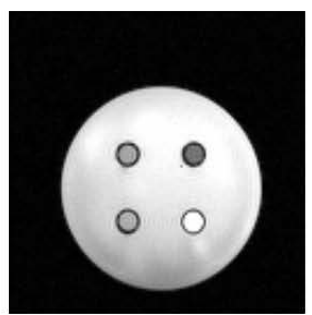

8250

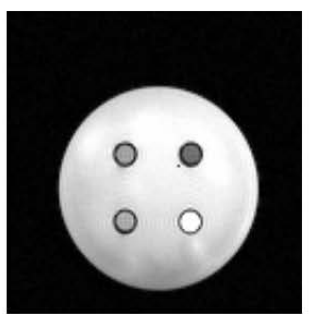

4375

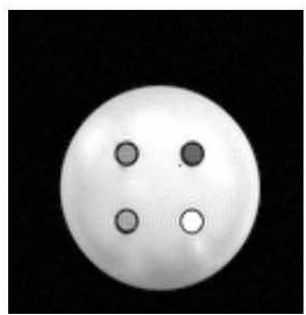

$500(\mathrm{~Hz})$

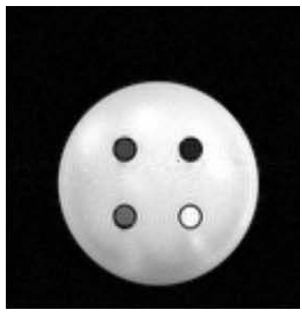

Figure 4. MT-phantom and MT-effects for $8 \%$ agar. Image quality and MT-effects depending on the frequency offsets (red: Agar 8\%). See also Table 1. 
Table 1. Signal intensities (mean, max, stdev) for the red marked region (8\% agar concentrate) in Figure 4 in dependence of the SAR-factor and the off-frequency range from 500 to $16000 \mathrm{~Hz}$.

\begin{tabular}{cccccc}
\hline SAR & $\boldsymbol{\Delta} \boldsymbol{f}=\mathbf{1 6 0 0 0}$ & $\mathbf{1 2 1 2 5}$ & $\mathbf{8 2 5 0}$ & $\mathbf{4 3 7 5}$ & $\mathbf{5 0 0}$ \\
\hline 1 & $204,239,15$ & $186,215,13$ & $180,206,14$ & $168,188,11$ & $57,74,11$ \\
2 & $217,247,14$ & $196,240,25$ & $197,244,26$ & $190,219,12$ & $89,135,21$ \\
4 & $213,283,25$ & $201,227,15$ & $216,271,25$ & $207,267,26$ & $118,187,25$ \\
\hline
\end{tabular}

(mean, max, std).

Table 2. Signal intensities (mean, stdev) and signal-to-noise ratios (SNR) for the red marked region (8\% agar concentrate) in Figure 4 at the frequency-offset of $500 \mathrm{~Hz}$ for three different sequence types: the new MT-SER-FLASH (2D), a standard 2D- and 3D-FLASH with MT-preparation.

\begin{tabular}{cccccccccc}
\hline \multicolumn{2}{|c}{ MT-SER-FLASH } & \multicolumn{3}{c}{ 2D MT-FLASH } & \multicolumn{3}{c}{ 3D MT-FLASH } \\
\hline Agar 8\% & Background & Ratio & Agar 8\% & Background & Ratio & Agar 8\% & Background & Ratio \\
\hline 115,16 & 8,5 & 14 & 128,12 & 7,4 & 18 & 82,12 & 2,1 & 41 \\
\hline
\end{tabular}

(mean, std).

\section{Results}

The MT-SER-FLASH is performed in 19 minutes rather than 1 hour and provides images of good quality (Figure 4). The SAR could be reduced to 69\% instead of more than $100 \%$ with a standard 3D-FLASH with MTpreparation. Optimal echo location (Figure 3) could be achieved in interleaved mode and a g-scaling of 42, 28, 14 for the three gradients G1 - $3_{\mathrm{d}}$ respectively. The MT-Effect depending on the SAR-Factor (red circle: Agar 8\%) is shown in Figure 4. It demonstrates (Table 1 ) that an adequate MT-effect compared to the SAR $=1$ case is only guaranteed for the SAR = 2 case. For the SAR = 2 case (full brain, $80 \mathrm{~kg}$ ) the exposition is $69 \%$ of the limit.

The signal-to-noise ratio (SNR) of the proposed MT-SER-FLASH (2D) in comparison to the SNR of a standard 2D-and 3D-FLASH with MT preparation at $500 \mathrm{~Hz}$ off-resonance-where the strongest MT-effect and the lowest SNR is to be expected-are summarized in Table 2. It shows that the SNR of the MT-SER-FLASH $(\mathrm{SNR}=14)$ is very similar to the 2D standard FLASH with MT preparation $(\mathrm{SNR}=18)$.

\section{Conclusion}

The net reduction of scan time and SAR enables the use of quantitative model based magnetization transfer imaging for more sophisticated purposes within a clinical environment.

\section{Acknowledgements}

This work was supported by the Ruth \& Arthur Scherbarth foundation, grant 2249.

\section{References}

[1] Pike, G.B. (1996) Pulsed Magnetization Transfer Contrast in Gradient Echo Imaging: A Two Pool Analytic Description of Signal Response. Magnetic Resonance in Medicine, 36, 95-103. http://dx.doi.org/10.1002/mrm.1910360117

[2] Sled, J.G. and Pike, G.B. (2000) Quantitative Interpretation of Magnetization Transfer in Spoiled Gradient Echo MRI Sequences. Journal of Magnetic Resonance, 145, 24-36. http://dx.doi.org/10.1006/jmre.2000.2059

[3] Sled, J.G. and Pike, G.B. (2001) Quantitative Imaging of Magnetization Transfer Exchange and Relaxation Properties in Vivo Using MRI. Magnetic Resonance in Medicine, 46, 923-931. http://dx.doi.org/10.1002/mrm.1278

[4] Kiefer, C., Brockhaus, L., Cattapan-Ludewig, K., Ballinari, P., Burren, Y., Schroth, G. and Wiest, R. (2009) Multiparametric Classification of Alzheimer's Disease and Mild Cognitive Impairment: The Impact of Quantitative Magnetization Transfer MR Imaging. Neuroimage, 48, 657-667. http://dx.doi.org/10.1016/j.neuroimage.2009.07.005

[5] Cercignani, M. and Alexander, D.C. (2006) Optimal Acquisition Schemes for in Vivo Quantitative Magnetization Transfer MRI. Magnetic Resonance in Medicine, 56, 803-810. http://dx.doi.org/10.1002/mrm.21003 
[6] Reese, T.G., Benner, T., Wang, R., Feinberg, D.A. and Wedeen, V.J. (2009) Halving Imaging Time of Whole Brain Diffusion Spectrum Imaging and Diffusion Tractography Using Simultaneous Image Refocusing in EPI. Journal of Magnetic Resonance Imaging, 29, 517-522. http://dx.doi.org/10.1002/jmri.21497

[7] Feinberg, D.A., Reese, T.R. and Wedeen, V.J. (2002) Simultaneous Echo Refocusing in EPI. Magnetic Resonance in Medicine, 48, 1-5. http://dx.doi.org/10.1002/mrm.10227

[8] Feinberg, D., Reese, T.G. and Wedeen, V.J. (2000) Simultaneous Image Refocusing (SIR): A New Approach to Multi-Slice MRI. Proceedings of 8th Scientific Meeting ISMRM, Denver, April 2000, 681.

[9] Feinberg, D.A., Moeller, S., Smith, S.M., Auerbach, E., Ramanna, S., Glasser, M.F., Miller, K.L., Ugurbil, K. and Yacoub, E. (2010) Multiplexed Echo Planar Imaging for Sub-Second Whole Brain FMRI and Fast Diffusion Imaging. PLoS One, 5, e15710. http://dx.doi.org/10.1371/journal.pone.0015710

[10] Gunther, M. and Feinberg, D.A. (2005) Simultaneous Spin-Echo Refocusing. Magnetic Resonance in Medicine, 54, 513-523. http://dx.doi.org/10.1002/mrm.20587 
Scientific Research Publishing (SCIRP) is one of the largest Open Access journal publishers. It is currently publishing more than 200 open access, online, peer-reviewed journals covering a wide range of academic disciplines. SCIRP serves the worldwide academic communities and contributes to the progress and application of science with its publication.

Other selected journals from SCIRP are listed as below. Submit your manuscript to us via either submit@scirp.org or Online Submission Portal.
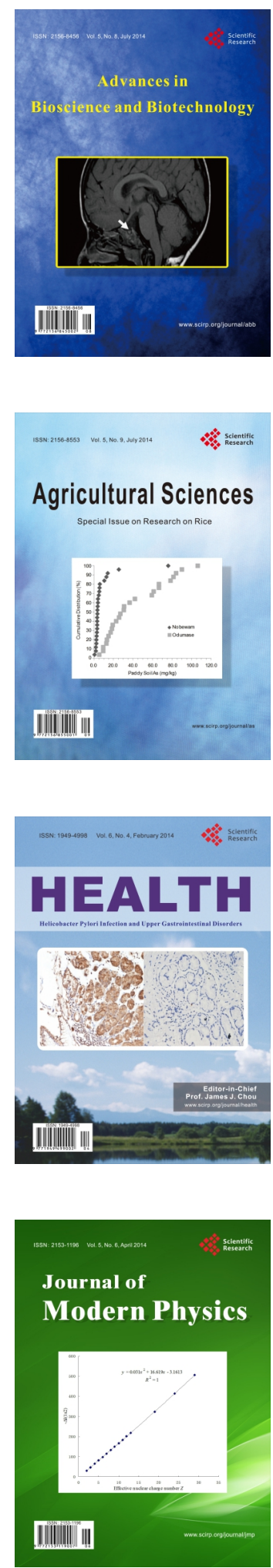
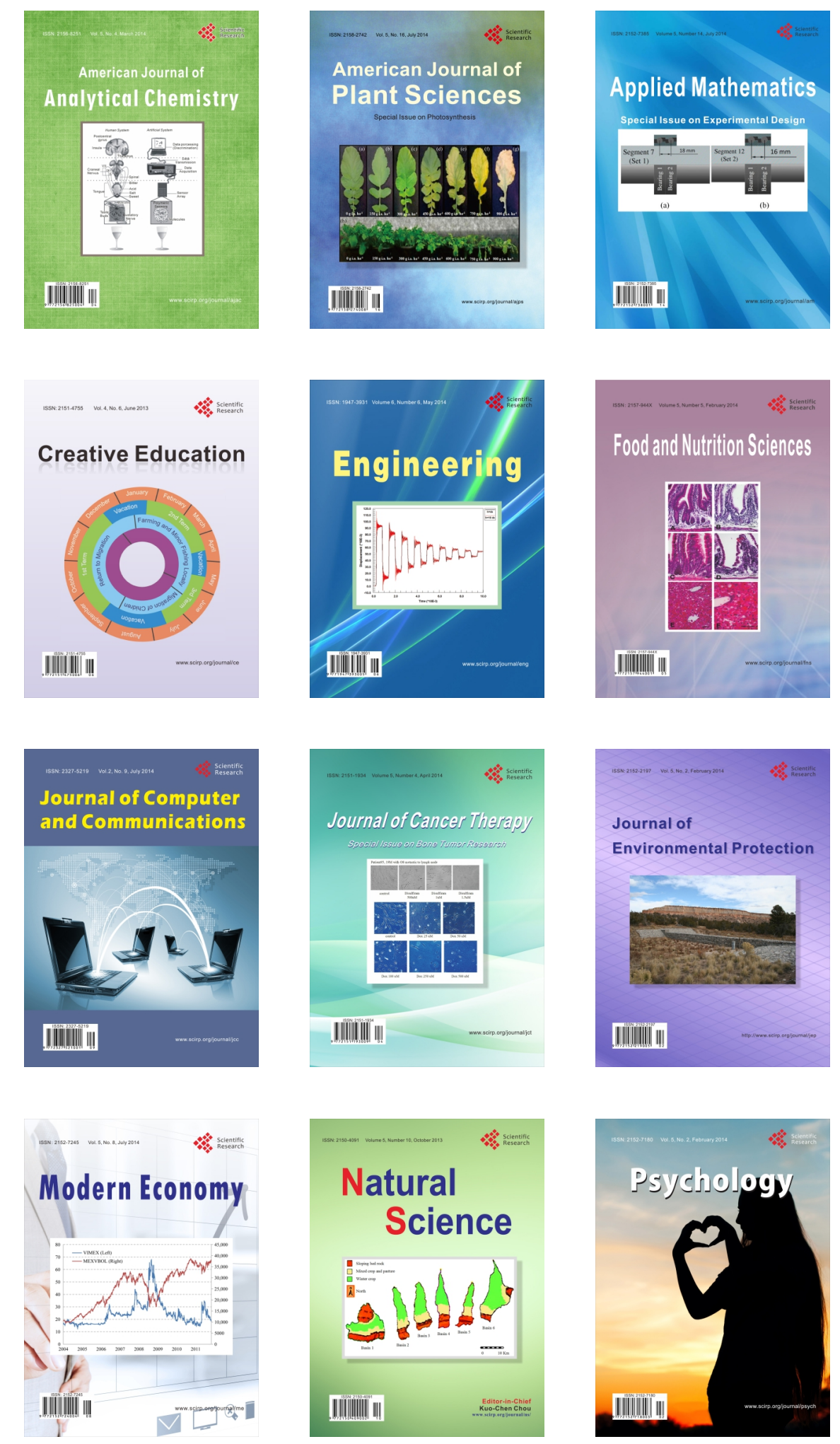